\title{
Man-machine Relationship and Acoustic Design of Portable Earphones for Youths
}

\author{
Liu Guo-qing \\ Wenzhou Vocational \& Technical College, Wenzhou. 325035 \\ 3685424@qq.com
}

Keywords: Design, Youths,Functional, Psychology.

\begin{abstract}
People oriented" design concept has become the mainstream and humanized, emotional design has become the trend of product design. Product design aims at serving human beings better as well as making life convenient and comfortable rather than simply design. The earphones I design in this paper mainly targets at the youth groups such earphones are fashionable and popularized among fitness enthusiasts and extreme sports enthusiasts.
\end{abstract}

\section{Introduction}

\subsection{Independence of consumption capacity of teenagers}

Perception, three-dimensional abstract thinking, judgment as well as adaptive capacity to surroundings all have reached the peak of growth during the period of youth. Their self-conscious development is becoming more perfect and thy gradually get rid of dependence on parents. As a result, compared with teenagers and children, the youth have gradually grown into independent policymakers with independent purchasing behavior, and autonomy consumption also begins. Simultaneously, the role of consumer choices and decisions of the youths is also growing in most household consumption. Parents gradually change the position with their kids and need their advice, so along with the purchase consequent. After participating in work, they have stable economic source. In addition, they have no economic burden at all, all these provide an important material foundation for their independent consumption and different individual demands.

\subsection{Influence of consumption capacity}

Social transformation, cultural variation as well as values change in the era of reform and opening up mainly reflect on the youth. During the development process, market economy, flowing commodity information as well as undulating market conditions are also firstly reflected in the consumption behavior of the youth. As a result, the consumer attitude of young people has an exerted great influence on other consumer groups. The youth often bear the brunt when a new and stylish product launching. Take a metaphor which is not so appropriate for example: the youth is "the first person to eat crab" in consumer market. The consumption of young people gradually influences and infects the affirmation of the new product of other consumer groups. Moreover, with the growing influence of the youth in family, young people are becoming the "second-generation owner" in household consumption. Meanwhile, with the marriage and having children of young people, after they build their own small families as well as they serve as the role of parents, they have become the "third generation of media people" in their household consumption, thus their buying behavior and attitudes are also direct affect the next generation. Practice has proved that the mother's eating habits will be first formed in memory of the children. And no other consumers can be compared with such a deep, subtle influence. Therefore, the striving for youth consumers can win the lifelong customers which can effect of two generations.

\subsection{Features of youth consumption}

1) They have strong consumption ability and huge market potential. When bringing huge impact on modern life, development of science and technology also plays a huge role. The richer economic incomes of the youth come from their innovation ability as well as knowledge updating advantage. In addition, they commonly have small family economic burden, they all hold strong desires and new 
ideas and focus on leisure and entertainment. As a result, the consumer group with the strongest consumption ability and the biggest market potential has become the feature of the youth.

2 ) Their consumption desires are strong with a contemporary and self-awareness. Youth consumer groups often show such a consumer psychology: I must own the goods that others don't have or only some people have, while if everyone owns it, I don't want it; which is a typical maverick, feisty, self-expression psychology.

3 ) Consumer behaviors are impulsive and full of emotion. Since the people in youth period are not completely matured coupled with limited experience, they can fully exert their personality which has not been fully finalized. Their rich inner and unrestrained characters lead more impulsive and less planned consumption. For example, more often, extrinsic factors such as shape, advertising, color, style, packaging of the product tend to be the primary factors in their decisions whether to buy the product or not. In addition, the consumption interests of youth consumers are of great randomness and volatility that their preferences are easily transferable from one product to another product, which reflects the emotional and impulsive of their consumption.

\section{Analysis of the current market products}

\subsection{Ordinary Headset}

All the earphones can't have the portability, so in addition to the portable models, there is the standard headset, this type is characterized by a larger cell size, and almost can wrap ears with thick frame structure, which is the exclusive indoor-use product.

\subsection{Ear-hook and neckband earphones}

Traditional headsets are of rich styles, but the design of head beam is a nightmare for the people with special hairstyle. Therefore, the appearances of ear-hook and neckband headphones solve the problem of hair. Of course, their strengths and weaknesses are the same with ear-hook earplugs that the sound leakage problem is very serious which is not resolved until today. Relatively common ear-hook earphones are the Sony EQ series, while neckband earphones are mainly sports headphones. Currently the market shares of both are not high, and the prices are also relatively cheap.

\subsection{Effect of headset volume}

Headset with greater volume has more developing directions; it can add more humane designs to make it more comfortable. The headset I design will not make people feel monotonous as well as mean and cheap on the whole. Apart from ensuring the big appearance and comfort feature, portability has become the issue we need to solve at the first time. The folding of headset on the market are designed for convenience only, while the folding style of headsets allow the destruction of the sense of beauty and design of headset itself. Therefore I designed a portable headset that is not only a portable headset, but also is not to abandon the beauty and sense of technology, of which there is a sense of fashion if young people hold it in hand, it's just same like woman carrying handbags that not just for convenience, it is clear that carrying handbags has become a fashion. And we young people, such as fitness enthusiasts and extreme sports enthusiasts, of course hold definitely a certain requirement of the appearance of the headsets that the headset must go into these things, let's say if there are two headsets, one is Chibi Maruko cartoon and another one is full of design sense, the choice for young people is very clear. Therefore headset is not only a tool, it has developed into a trend.

\section{Description of overall design scheme}

\subsection{Design purpose of portable headset}

Because modern headset is usually used in a single desktop computer for a long time and in normal circumstances, it seems that the users equip exclusive device for their computers after purchasing the headset. While although the headset us easy to carry, its sound effect is far worse than headphone which cannot meet the requirements of the youth as well as fashion group. In addition, long-term use of in-ear headset will greatly affect the hearing and cause inconvenience to life. As a result, an easy 
carrying and convenient headset can obtain the market, and the hand-printed rendering is shown in figure 1.
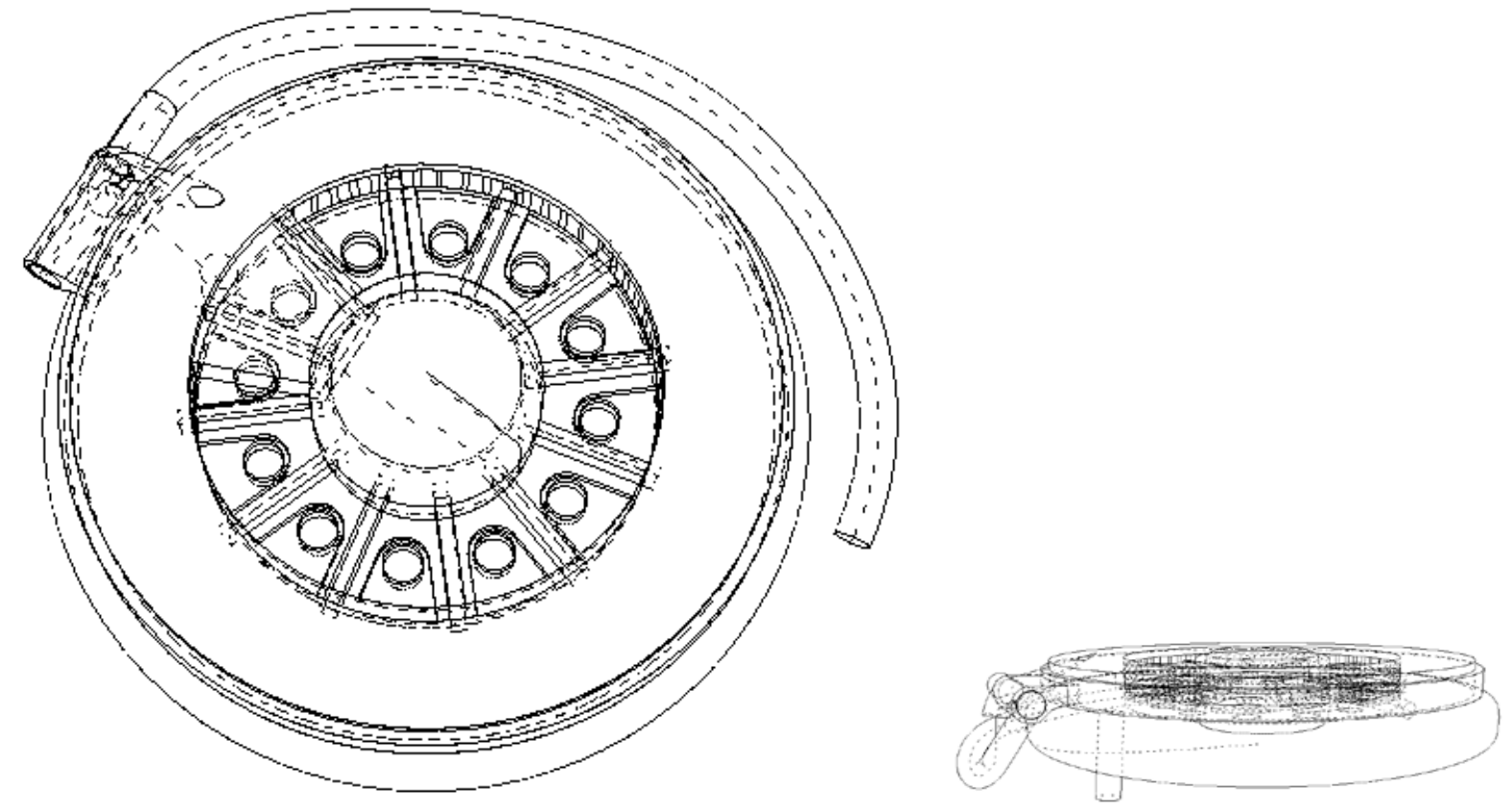

Figure 1 Hand-painted rendering

\subsection{Effect diagram of computer-aided product}

The earmuff must be soft and comfortable, and it is mostly filled with sponge inside and material outside uses leather or velvet. The material that this earmuff uses decides the absorption size of middle and high frequency, ears and diaphragm are separated for a distance to form a chamber between earphone and your head. Large round earmuff usually has a huge space inside, the sound acts on ears to form a quite good sense of space. A good headset should fully consider the effect of earmuff. As a result, the earmuff of mid-range and high-end headset cannot be freely replaced. Sounding unit is the most complex part with the highest technical content in headset design which determines the sound quality.

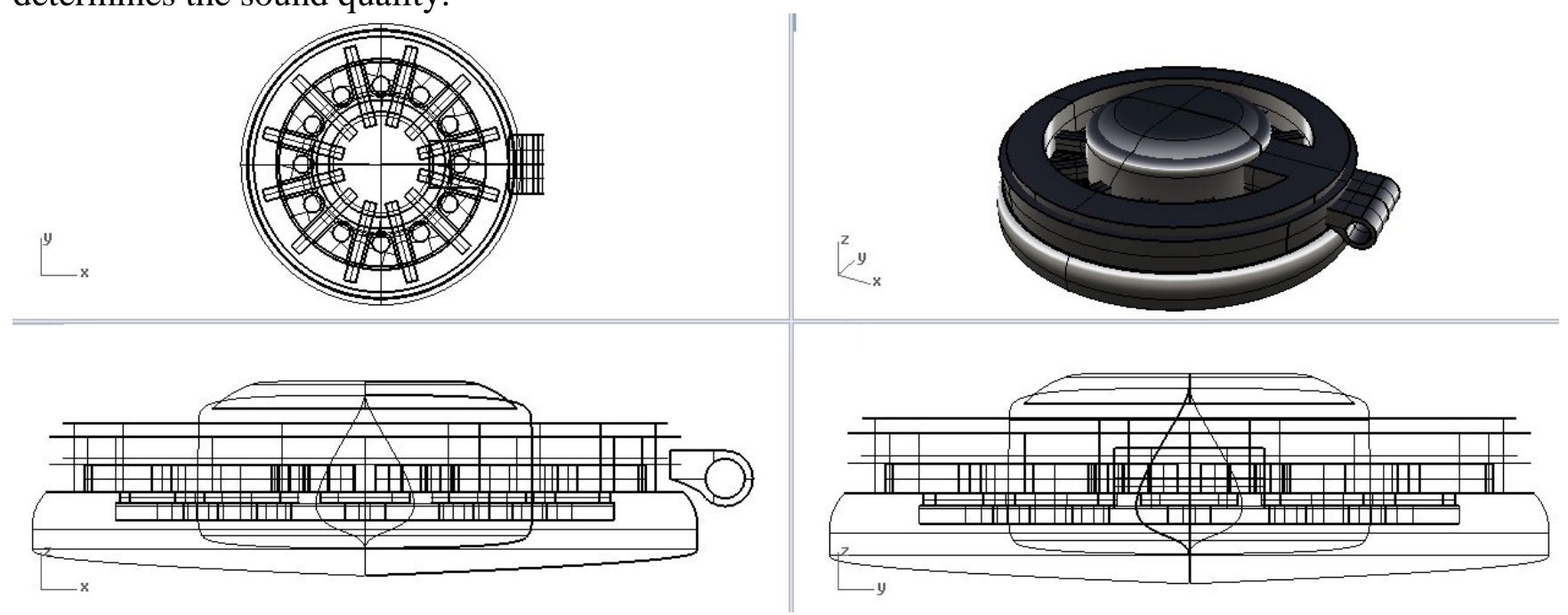

\subsection{Product details analysis}

Figure 2 Computer-aided effects

The link of headphones adopts metal which makes it more strong and durable. And at the same time, metallic luster is better than the clinker material and the earphones do not look cheap. After rotary folding, the sound emitting unit with rich scientific and technological sense will present outside completely and not cocoon the things inside any longer. Following is the main materials selection: the outer layer adopts frosted metal or polished metal and the sound emitting unit adopts 
plastic material, while spray painting makes it present metal texture. The reason I give up using mental is the weight, the cortex outside of ear hook is filled with sponge and all the connecting parts are mental. As shown in figure 3 and 4, the wireless designed blue-tooth connection allows the sports group use without any barrier.

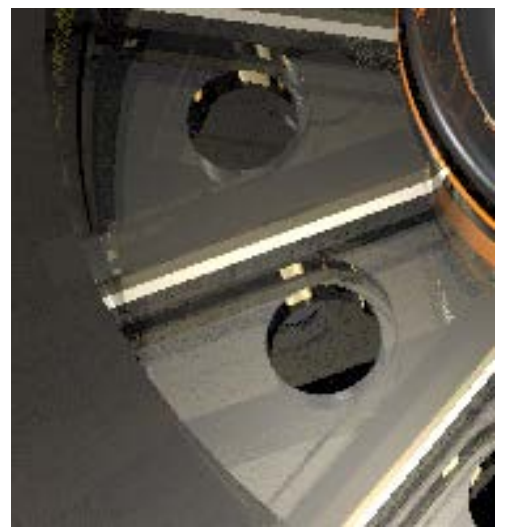

Figure 3 Detail display

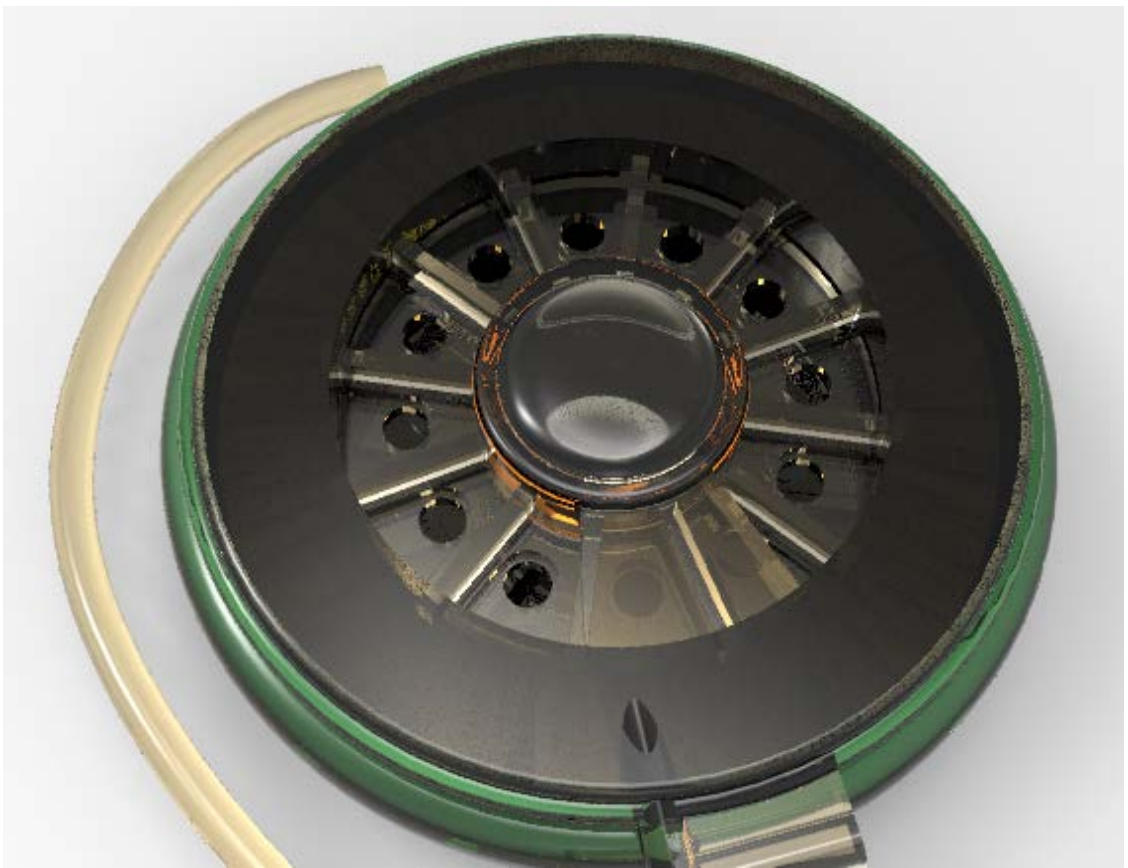

Figure 4 Material application

\section{Summary}

The emergence of a good product need to fully understand all the relevant information, accuracy of product positioning, freshness of product innovation, rationality of materials and colors, limitation of product design as well as promotion after putting into the market in the future.

\section{References}

[1] RUI Yan-nian.Innovation Theory and Application[M].Beijing: High Education Press, 2007.

[2] ZHANG Zhi-yuan, HE Chuan.Invention and Creation Methodology[M].Chengdu: Sichuan University Press, 2003.

[3] WANG Liang-shen, SUN Feng-hua.TRIZ Innovation Theory and Application Principles[M].Beijing: Science Press, 2010.

[4] WANG Ting-ting, LIU Xiao-xia.The Packaging Design of Vibration Feeding Equipment Based on the Finite Element Analysis[J].Packaging and Food Machinery, 2012, 30 (1) : 68-71.

[5] HOU Xiang-yang.Design of Packaging Cost : Applied Case of Deongaree Company in CPS[J].Packaging World, 2008 (1) : 49-50.

[6] Heilongjiang Science and Technology Hall.Theory of Inventive Problem Solving[M].Harbin: Heilongjiang Science and Technology Press, 2008.

[7] LYU Xin.Research on Product Innovation Design Based on 39 Contradictions Matrices and 40 Innovation Principles of TRIZ [J].Packaing Engineering, 2009，30（5）: 27-30. 